\title{
Gegenstand der Arbeit und ideologische Kontextualisierung
}

\subsection{Gegenstand der Untersuchung und relevante Begrifflichkeiten}

Am Anfang der vorliegenden Studie stand ein Dokument in Rollenform aus der Zentralbibliothek Zürich, auf das ich im Jahr 2002 aufmerksam geworden bin (Ms. or. 20 ). ${ }^{1}$ Es misst $4.7 \times 308 \mathrm{~cm}$ und war für mich ursprünglich ein Unikat. Das Dokument beeindruckt durch die darauf feststellbare Kombination von Textelementen und graphischen Gestaltungsmitteln. Seine Stellung als Einzelstück und Kuriosum hat dieses Exemplar rasch eingebüsst, liessen sich doch allein in westlichen, zumeist europäischen, wissenschaftlichen Institutionen zusätzliche Dokumente in grösserer Zahl belegen, die in unterschiedlicher Hinsicht Ähnlichkeiten mit dem Zürcher Belegstück aufweisen. $^{2}$

Die vorliegende Arbeit stützt sich auf ein Korpus, das etwa 120 Belegstücke umfasst. ${ }^{3}$ Dies lässt vermuten, dass das Anfertigen von Rollen von der hier untersuchten Art im islamischen Kulturraum früher, wenn auch nicht üblich, so doch weit verbreitet war. Die vorliegende Arbeit stützt sich aus naheliegenden Gründen allerdings nur auf jene Überbleibsel dieser Tradition, die durch glückliche Umstände bis heute erhalten geblieben sind. Es versteht sich von selbst, dass diese in der bisherigen Forschung noch kaum beachteten Dokumente hier nicht in globo aufgearbeitet werden können.

1 Vgl. Nünlist, Handschriften 74-8o (Ms. or. 20).

2 Diese Arbeit stützt sich nahezu ausschliesslich auf Belegstücke aus westlichen, v.a. europäischen Institutionen. Suleman, Introduction $2 \mathrm{f}$. (in Suleman, Word of God), macht darauf aufmerksam, dass gerade Koranhandschriften in der islamischen Welt für westliche Forscher schwer zugänglich seien. Verschiedene hier untersuchte Belegstücke enthalten den gesamten Korantext.

3 Vgl. die Übersicht der identifizierten Dokumente im Anhang (Appendix).
Diese Untersuchung konzentriert sich vielmehr auf ausgewählte Belegstücke.

Rollen können grundsätzlich als volumen oder als rotulus konzipiert werden. ${ }^{4}$ Beim volumen verlaufen die Textstellen horizontal zur Rollenrichtung. Auf einem rotulus sind die Zeilen mit dem Text jedoch in einem rechten Winkel zur Rollenrichtung kopiert worden. Da die hier untersuchten Belegstücke oft zugleich horizontal, vertikal und sogar kreisförmig ausgerichtete Textstellen enthalten, erweist sich eine Unterscheidung zwischen volumen und rotulus als wenig sinnvoll. ${ }^{5} \mathrm{Die}$ in dieser Untersuchung vorgestellten Dokumente werden einfach als Rollen bezeichnet. Derartige Belegstücke werden im islamischen Kulturraum heute gemeinhin țūmār genannt (von Griechisch tó tomárion).

Im Zentrum dieser Untersuchung stehen zwar die Dokumente in Rollenform selbst. Allerdings ist bei dieser Gelegenheit doch in allgemeiner Form darauf aufmerksam zu machen, dass diese Belegstücke zumeist in einem Behältnis aufbewahrt worden sind. Diese Behältnisse können aus Metall, ${ }^{6}$ Holz, ${ }^{7}$ Stoff,, ${ }^{8}$ Leder ${ }^{9}$ oder einem andern

4 Vgl. für die folgenden Ausführungen Gacek, Arabic Manuscripts 224-226 (Artikel Roll).

5 Gacek, op. cit. 225, meint, dass volumina stricto sensu in der islamischen Welt gänzlich unbekannt seien.

6 Vgl. dazu св 542 (Fondation M. Bodmer, Cologny-Genève); Badische Landesbibliothek, Karlsruhe, Hs RA 204, aus der Karlsruher Türkenbeute (siehe: http://www.tuerkenbeute .de/sam/sam_sch/RA204_de.php, Stand 17. August 2017); Bayerische Staatsbibliothek, München, Cod. arab. 2616 (bis anhin nicht beschrieben; siehe dazu Kapitel 7.2, vor und mit Anm. 38); Bodleian Library, Oxford: Ms. Arab. g 5 (R) (Behältnis aus Gold).

7 Zentralbibliothek Zürich: Ms. or. 2o; das Dokument wurde in einer gedrechselten Holzkapsel aufbewahrt.

8 Universitätsbibliothek Leipzig: B. or. 322 (https://www .islamic-manuscripts.net/content/index.xml: Abb. 24, Stand 17. August 2017).

(C) TOBIAS NÜNLIST, 2020 | DOI:10.1163/9789004429154_002

This is an open access chapter distributed under the terms of the CC BY-NC-ND 4.o license. 
geeigneten Material hergestellt worden sein. Sie sind zumeist mit einer Vorrichtung (Kette, Stoffbändel) versehen, die es erleichterte, diese Rollen am Körper zu tragen. Damit die Dokumente überhaupt in diese Behältnisse passten, mussten sie äusserst satt aufgerollt werden. Geht man beim Herausnehmen nicht mit der angezeigten Sorgfalt vor, können sich die Exemplare mit grosser Geschwindigkeit von selbst entrollen. Aus konservatorischen Gründen werden diese Rollen heute zumeist ausserhalb ihrer sehr engen Behältnisse aufbewahrt. ${ }^{10}$ Die in die Herstellung involvierten Kunsthandwerker haben diese hohe Spannung der Rollen aber mit grosser Wahrscheinlichkeit bewusst herbeigeführt.

Es ist zwar davon auszugehen, dass im Fall der soeben angeführten Belegstücke, Behältnis und Rolle von Anfang an zusammengehörten. ${ }^{11}$ Allerdings lässt sich dies nicht immer mit letzter Sicherheit beweisen. Es ist gut möglich, dass eine ältere Rolle in ein jüngeres Behältnis gesteckt wurde oder vice versa. Gerade Behältnisse aus Metall blieben länger erhalten als die Dokumente aus Papier selbst. Jedenfalls besitzt das Bernische Historische Museum eine Reihe von solchen Behältnissen; sie seien allerdings alle leer. ${ }^{12}$ Aus den angeführten Gründen werden diese Behältnisse in der vorliegenden Arbeit nur am Rand behandelt. Sie würden jedoch eine eigenständige Untersuchung verdienen. ${ }^{13}$

9 Staatsbibliothek Berlin: Ms. or. oct. 146 (http://digital .staatsbibliothek-berlin.de/suche? category $\%{ }_{5} \mathrm{~B} \circ \% 5 \mathrm{D}=\mathrm{I}$ slamische $\% 20 H a n d s c h r i f t e n \& q u e r y S t r i n g=146 ; \quad$ ohne Abbildung des Behältnisses. Stand 17. August 2017).

10 Das wiederholte Entrollen und Aufrollen der Dokumente führt leicht zu Einrissen im Papier. Ausserdem lösen sich bei den Rollbewegungen die Farbpigmente viel eher vom Beschreibstoff als dies bei handschriftlichen Dokumenten in Kodexform der Fall ist. Vgl. die in Anm. 6-9 angeführten Exemplare.

12 F. Linder Mathieu, Bernisches Historisches Museum (3. April 2009).

13 Mit der Frage hat sich ansatzweise befasst Schienerl, Rechteckige Amulettbehältnisse; ders., Ägyptische Amulettbehältnisse.
Das Rollenformat ist für das Anfertigen schriftlicher Dokumente in der islamischen Welt gar nicht so selten, wie es auf den ersten Blick scheinen mag. ${ }^{14}$ Jedenfalls ist festzuhalten, dass es neben der dominanten Stellung der Kodexform im islamischen Kulturraum nie gänzlich unbekannt war und sich letztlich auch aus Traditionen herleiten lässt, die im Nahen Osten bereits vor dem Aufkommen des Islams beheimatet waren. Allgemein bekannt ist die grosse Bedeutung von Thorarollen im Judentum. Verträge und weitere Dokumente juristischen Inhalts wurden später auch in islamischen Kontexten häufig als Rollen angefertigt. Es ist daher sinnvoll, jene äusseren (formalen) und inneren (inhaltlichen) Kriterien festzuhalten, die für die Berücksichtigung der ausgewählten Dokumente im Rahmen der vorliegenden Arbeit ausschlaggebend waren. Die in dieser Studie untersuchten Dokumente sollen die folgenden äusseren Kriterien erfüllen:

\begin{tabular}{|c|c|c|}
\hline & Einschluss & Ausschluss \\
\hline Format & Rolle (țūmār $)$ & Kodex \\
\hline Schrift & Handschriftlichkeit & $\begin{array}{l}\text { Drucke, auch } \\
\text { Blockdrucke } \\
\left(\text { Tarš-Technik }^{15}\right)\end{array}$ \\
\hline Beschreibstoff & Papier & Pergament, ${ }^{16}$ Papyrus \\
\hline Gestaltung & $\begin{array}{l}\text { Kombination } \\
\text { textlicher und } \\
\text { graphischer } \\
\text { Elemente }\end{array}$ & Text allein \\
\hline Farbe & Mehrfarbigkeit & Einfarbigkeit ${ }^{17}$ \\
\hline
\end{tabular}

Neben den soeben zusammengestellten formalen Kriterien spielten auch inhaltliche Aspekte bei der

14 Vgl. dazu auch Gacek, Arabic manuscripts 224-226 (Artikel Roll).

15 Dokumente in Tarš-Technik sind von K.R. Schaefer in einer früheren Studie untersucht worden (Enigmatic charms). Vgl. dazu ausführlicher unten bei Anm. 66-67.

16 Vgl. dazu unten nach Anm. 40 und bei Anm. 51-53; auch Kapitel 7.2 bei Anm. 38-42.

17 Vgl. z. B. Thüringer Universitäts- und Landesbibliothek, Jena, Ms. Prov. o. 225a (beschrieben von Sobjeroi, Handschriften 54f., Nr. 26). Ausserdem macht Ory, Un 
Auswahl der untersuchten Belegstücke eine Rolle. Während die inhaltlichen Einschlusskriterien erst im Lauf der weiteren Ausführungen verdeutlicht werden, lassen sich die Ausschlussgründe bereits an dieser Stelle festhalten. Die vorliegende Arbeit berücksichtigt demnach keine Dokumente folgenden Inhalts:

a. Pilgerfahrtsrollen bzw. Pilgerfahrtszertifikate ${ }^{18}$

b. Kalender (taqwim) in Rollenform ${ }^{19}$

c. Erlasse von Sultanen und Verwaltungsstellen $(\text { firmān, berāt })^{20}$

d. Besitzurkunden ${ }^{21}$

nouveau type de mușhaf, auf weitere einfarbige Rollen aufmerksam.

18 Aus der Ayyubidenzeit (1171-1252) sind auch Pilgerfahrtszertifikate in Rollenform bekannt; vgl. Sourdel, Certificats de pèlerinage; vgl. dazu auch Anm. 3 .

19 Vgl. z. B. Heidelberg, Universitätsbibliothek, Cod. Heid. Orient. 14; Maddison und Savage-Smith, Sciences, tools and magic. Part I: Body and spirit 28of. (Nr. 170-172).

Vgl. Derman, Siegel des Sultans 166-181 (jeweils mit Abbildungen). Bereits die Mamluken haben Verträge auf Rollen festgehalten (14. Jh.; Diskussion mit Dr. D. Potthast, München, in Zürich, 23. Februar 2018). Siehe auch Potthast, Drei Fragmente 37of., 403, 406f., $411 \mathrm{f}$. Die Rollen P.Aragón 145-152 und 163-164 sind im 14. Jh. angefertigt worden. Siehe z.B. Archivo de la Corona de Aragón, ACA, COlecciones, Cartas árabes, núm. 147 (http:// pares.mcu.es/ParesBusquedas/servlets/Control_servlet ?accion $=3 \&$ txt_id_desc_ud $=4800512 \&$ fromagenda $=I \& t$ xt_primerContiene $=1$; Stand 3. Februar 2018).

Die Besitzungen der Heiligtümer in Ardabīl und Mašhad in Iran wurden im 18. Jh. auch auf Rollen festgehalten:

Ardabīl: Muhammad Qāsim Beg ordnete 1207/1793 das Erstellen einer Liste mit den damaligen Besitzungen des Schreins an. Mullāh Muhammad Țāhir Mustawfì bereitete darauf eine Rolle vor, die $12 \times 757 \mathrm{~cm}$ misst. Diese Rolle wurde unter dem Titel The Treasure of Sheikh Safi al-din durch die Nationalbibliothek in Tabriz als kleines Buch veröffentlicht (siehe Iranian Cultural Heritage, Handicrafts and Tourism Organization, Sheikh Safi alDin 181).

Mašhad: Im 18. und 19. Jh. wurden zwei Rollen angefertigt, die die Besitzungen des waqf des Āstān-i Qudsi Radawī aufzählen: a. die Rolle des 'Alī Šāh Afšār (Stiftungs- und Besitzurkunde; datiert 116o/1747), Signatur: 111; b. die Rolle (Stiftungsurkunde) des 'Aḍud alMalik (1273/beginnt September 1856), Signatur 112. Für weitere Informationen und Abbildungen siehe https:// www.imamreza.net/old/eng/imamreza.php?id=5118 und https://razavi.aqr.ir/portal/home/showpage.aspx?o e. Genealogien (Herrscher-Genealogien, ${ }^{22}$ SufiSilsilas ${ }^{23}$ )

f. Sufi-Iğāzas ${ }^{24}$

g. Gedichtsrollen (z.B. picture poems von Ğilyānī $)^{25}$

h. Liturgierollen (aus christlichen Kontexten) ${ }^{26}$ Bei der Herstellung von Rollen von der hier berücksichtigten Art wurden mehrere Streifen Papier aneinander geklebt. Die Breite dieser Streifen misst zwischen ca. 4.0 und $12.5 \mathrm{~cm}$. Ihre Länge variiert zwischen ca. 30 und $50 \mathrm{~cm}$. Gerade die Breite der verwendeten Streifen und damit der Rollen selbst lässt Rückschlüsse auf Entstehungszeit und Entstehungsort zu. Auch die Wahl der eingesetzten graphischen Gestaltungsmittel oder das Vorhandensein bestimmter Textelemente lassen sich in Hinblick auf eine Typologisierung beiziehen. Diese Kategorisierung gestattet eine bessere Ori-

bject $=$ relatedtopics $\&$ categoryid $=2$ b68b2c1-e 4 b6 $-4472-$ b1 d1-1e3be5cod5e9\&webpartid=8ac1495c-b998-4eo7-9e3e -diofod43cbob\&id=8933adcb-7851-434a-bff9-69437196a 65 e (Stand beide 31. Januar 2018).

22 Für Herrscher-Genealogien in Rollenform vgl. Zentralbibliothek Zürich, Ms. or. 21 und 22-23 (beschrieben in Nünlist, Handschriften 8o-84).

23 Für eine Sufi-Silsila in Rollenform vgl. Heidelberg, Universitätsbibliothek, Cod. Heid. Orient. 487 (aus dem Orden der Aḥmadiyya-Badawiyya).

24 Vgl. z.B. Ms. or. fol. 1622 (Staatsbibliothek zu Berlin).

25 'Abd al-Mun'im b. 'Umar al-Ǧilyānī (531-6o2/1136-12o6) stammt ursprünglich aus Spanien, war Sufi und lebte zur Zeit der Ayyubiden in Damaskus. Er verfasste sogenannte picture poems (vgl. Julia Bray, Oxford). Davon sind mehrere Handschriften erhalten geblieben (am wichtigsten: Arabic 69o, John Rylands Library, Manchester; datiert gemäss Kolophon Bl. 117a: 27. Ramaḍān 735/21. Mai 1335; es handelt sich um eine Abschrift eines älteren Exemplars, das noch von Ǧilyānīs Sohn angefertigt worden war). Das Exemplar aus Manchester und weitere Handschriften haben zwar Kodexform. J. Bray konnte aber aufzeigen, dass jeweils mehrere Seiten daraus eine Einheit bilden, die eine Länge von ca. $2-3 \mathrm{~m}$ aufweisen und nur zusammen sinnvoll gelesen werden können. J. Bray hatte dazu einen Workshop organisiert (Oxford, 23--24. Juli 2015). Vgl. auch al-Ǧilyānī, Dīwān atTadbìg.

26 Vgl. dazu z. B. Gerstel, Liturgical scrolls; Maniaci, Liturgical scroll; Bokotopulos, Byzantine illuminated manuscripts. 
entierung in der beträchtlichen Materialfülle. Wie jede andere Einteilung ist aber auch sie mit dem Nachteil verbunden, dass sie unscharf ist.

Die Belegstücke wurden in einem ersten Schritt in drei nach regional-sprachlichen Kriterien definierte Gruppen eingeteilt. ${ }^{27}$ Es wurde demnach ganz grob unterschieden zwischen $\mathrm{A}$. einem Städtedreieck-Typ, der in erster Linie mit der Region zwischen Konya, Tabriz und Bagdad in Verbindung zu bringen ist und dessen Belegstücke zumeist aus dem 14. und 15. Jh. stammen; B. einem persischen Typ und C. einem osmanischtürkischen Typ.

Die weiteren Ausführungen verdeutlichen diese Dreiteilung in einem ersten Schritt anhand von drei Dokumenten aus Schweizer Institutionen, die sich auch in einem internationalen Kontext durch ihre hohe Qualität auszeichnen. Sie werden hier als Leitdokumente für die drei angeführten Typen herangezogen. Die aus den Auseinandersetzungen mit weiteren Dokumenten aus europäischen Institutionen abgeleiteten Erkenntnisse beeinflussten aber die vorgenommene Dreiteilung massgeblich. Bei den vorerst näher vorzustellenden Exemplaren bzw. Typen handelt es sich um:

A. M III 173, Universitätsbibliothek Basel. Dieses Exemplar vertritt den Städtedreieck-Typ. ${ }^{28}$ Mit diesem Typ befasst sich Kapitel 4.1-10.

B. Св 542, Fondation M. Bodmer, ColognyGenève. Es steht für den persischen Typ. Kapitel 5.1-5 stellt Belegstücke dieser Art vor.

27 Vgl. dazu Nünlist, Entzauberte Amulettrollen; dieser Aufsatz erschien zwar erst 2019. Allerdings handelt es sich eigentlich um den frühesten Beitrag, den ich im Rahmen meiner Auseinandersetzung mit diesen Dokumenten in Rollenform verfasste.

28 Für eine Abbildung der ganzen Rolle siehe: http://w ww.e-manuscripta.ch/doi/10.7891/e-manuscripta-16628 (Stand 20. August 2017). Vgl. die Beschreibung in Schubert und Würsch, Arabische Handschriften 243-246 (Nr. 101). Dieses Dokument wurde bei der kodikologischen Erfassung ins 17.-18. Jh. datiert. Diese chronologische Einordnung ist deutlich zu korrigieren. Gestützt auf Vergleichsdokumente ist eine Entstehung am Ende des 14. bzw. am Anfang des 15. Jh. zu vermuten. Vgl. die Beschreibung Kapitel 4.8 .
C. Ms. or. 20, Zentralbibliothek Zürich. Dieses Belegstück wird hier als Leitdokument für den osmanisch-türkischen Typ vorgestellt. ${ }^{29} \mathrm{Kapi}$ tel 6 widmet sich der näheren Untersuchung dieser Art von Dokumenten.

Die folgenden Ausführungen halten die auffälligsten Merkmale des jeweiligen Typs fest. Sie stellen den drei Gruppen und ihren Leitdokumenten zusätzliche Belegstücke zur Seite und objektivieren die dreifache Gliederung dadurch.

A. Städtedreieck-Typ (Dreieck mit den Eckpunkten Konya, Tabriz, Bagdad und der weiteren Region darum herum, gerade in Richtung Zentralasien): Das auffälligste Merkmal dieses Typs besteht darin, dass ein zumeist mehrteiliges Textband die Rolle auf ihrer gesamten Länge oder einem bedeutenden Teil davon umfährt. In diesen seitlichen Schriftbändern wurde der Text vorwärtsoder rückwärtslaufend, jedenfalls aber in Rollenrichtung, kopiert. Der Text zwischen den beiden seitlichen Schriftbändern wurde zumeist auf horizontalen Zeilen notiert. In den Beschreibungen der einzelnen Belegstücke wird dieser von den Seitenbändern eingefasste Teil der Rolle als Mittelstreifen bezeichnet.

Belegstücke dieses Typs sind relativ alt und datieren zumeist aus dem 14.-15. Jh. ${ }^{30}$ Bei der

29 Nünlist, Handschriften 74-8o (Ms. or. 20).

30 Im Rahmen der vorliegenden Untersuchung liessen sich keine Exemplare identifizieren, die vor 1300 entstanden sind. Es ist allerdings unwahrscheinlich, dass im 14. Jh. plötzlich und wie aus dem Nichts heraus Dokumente von jener hohen Qualität angefertigt wurden, die in dieser Arbeit vorgestellt werden. Die Tradition dürfte vielmehr bereits zuvor gut ausgebildet gewesen sein. Entsprechende Belegstücke sind aber soweit ersichtlich nicht erhalten geblieben.

Ory, Un nouveau type de mușhaf, beschreibt einen Bestand von 44 Koranfragmenten in Rollenform aus dem 7.-12. Jh. Beim Beschreibstoff handelt es sich mehrheitlich um Pergament (29 Hs.); 15 Belege sind auf Papier kopiert worden. Diese Rollen wurden zusammen mit Pilgerfahrtszertifikaten und einigen Archivdokumenten in der Omeyyaden-Moschee in Damaskus aufbewahrt. Sie wurden in Anschluss an einen Brand 1892-1893 entdeckt und von den Osmanen nach Istanbul gebracht (heute im Türk ve İslam Eserleri Müzesi). Die Pilgerfahrtsdokumente sind von D. Sourdel studiert worden: Certificats de 
Herstellung wurde ein starkes Papier verwendet; es ist oft auf ein textiles Gewebe aufgezogen. Die Breite dieser Dokumente beträgt ca. $12 \mathrm{~cm} .^{31}$ Ihr hohes Alter erklärt, dass sie häufig nur fragmentarisch erhalten geblieben sind. Das längste bis anhin nachgewiesene vollständige Dokument erreicht bei einer Breite von $11.9 \mathrm{~cm}$ eine Länge von $15.72 \mathrm{~m} .{ }^{32} \mathrm{Im}$ Fall verschiedener fragmentarisch erhaltener Belegstücke (z.B. M III 173, Basel; Is 1624 , Dublin) lässt sich vermuten, dass ihre ursprüngliche Länge $12 \mathrm{~m}$ erreicht oder sogar überstiegen hatte. Noch länger ist mit 20 Metern nur ein weiteres Belegstück; es ist allerdings wohl erst im 17. Jh. entstanden. Auch sind die Arbeiten daran nicht abgeschlossen worden. ${ }^{33}$

pèlerinage (vgl. bei Anm. 18). Darin fehlen Hinweise, dass die Koranfragmente seit ihrer ersten Bearbeitung durch S. Ory weitere Aufmerksamkeit erhalten haben. Zu diesen Damaszener Dokumenten siehe auch Bauden, Mamluk era documentary studies v.a. 53 f.; Ory, Un nouveau type de muṣhaf, Tafeln I-IV; weitere Abbildungen in alMunağğid, al-Hatț al-'arabī 9o-95.

Auch Schaefer, Enigmatic charms, macht unter den von ihm erfassten Belegstücken in Tarš-Technik auf Dokumente aufmerksam, die vor 1300 entstanden sein dürften; siehe op. cit. 44 (Exemplar aus Strassburg); zur Frage der Datierung dieser Tarš-Dokumente siehe bei ihm ebenso $41^{-} 5^{1}$ (Kapitel Calligraphy and the issue of dating the Arabic block print). Sehr oft werden diese Blockdrucke in der Literatur ins 10.-14. Jh. datiert.

31 Prof. F. Bauden (Liège) machte mich darauf aufmerksam, dass das in den mamlukischen Kanzleien zur Anfertigung von amtlichen Dokumenten verwendete Papier ca. $50 \times 50 \mathrm{~cm}$ mass. Beim Zuschneiden des Papiers für das Anfertigen der Rollen ergab sich eine Breite von ca. $12.5 \mathrm{~cm}$, wenn man vier Streifen herstellte. Die Übereinstimmung mit der Breite der Rollen dieses Typs ist auffällig (persönliche Unterhaltung, Cambridge, September 2013).

32 Dieses Dokument misst $11.9 \times 1572 \mathrm{~cm}$, datiert aus dem Jahr 754/1353-1354 und wurde als Lot 127 (Qur’an scroll, Art of the Islamic and Indian Worlds) bei Christie's, London, zur Versteigerung am 5. Oktober 2010 angeboten, aber wieder zurückgezogen (http://www.invaluab le.com/auction-lot/quran-scroll-127-c-ce28dc7ef3; Stand 17. August 2017); vgl. dazu Kapitel 4.10.

33 Es handelt sich um B. or. 322 (Universitätsbibliothek Leipzig): Dieses Belegstück ist aber wohl im 17. Jh. angefertigt worden. Das Dokument misst $12.7 \times 2003 \mathrm{~cm}$ und ist zusammen mit einem Stoffetui erhalten geblie-
Im Fall dieses Typs lassen sich einfacher und komplexer gestaltete Subtypen feststellen:

A.1: Bei der einfachsten Form dieses Typs fasst das Textband die gesamte Rolle seitlich ein und schliesst sie am Ende ab. ${ }^{34}$ Es kann aber auch sein, dass die beiden seitlichen Schriftbänder zumindest einen bedeutenden Teil der Rolle einfassen. ${ }^{35}$

A.2: Es lassen sich überdies Belegstücke nachweisen, auf denen ein mehrteiliges Schriftband unterschiedliche Figuren (Kreise bzw. Medaillons; an ihren beiden Enden abgerundete Rechtecke) umfährt. Die Schriftbänder verbinden die aufeinanderfolgenden Figuren dabei nicht untereinander. Dies ist auf einem Exemplar aus Kopenhagen besonders ausgeprägt der Fall. Das Schriftband ist in diesem Fall dreiteilig. ${ }^{36}$

A.3: Die dritte Unterform dieses Typs weist einen besonders komplexen Aufbau auf. Auf Dokumenten dieser Art dominiert ein Knotenband. Ein mehrteiliges Schriftband wechselt unter Umfahrung von Medaillons bzw. Knoten von der linken auf die rechte Seite der Rolle bzw. vice versa. Die Schriftbänder überschneiden sich dabei. Diese Übergangsstellen sind sorgfältig gestaltet. ${ }^{37}$

B. Der persische Typ: Sein auffallendstes Merkmal besteht darin, dass auf diesen Dokumenten

ben. Vgl. dazu Fleischer, Catalogus $55^{2}$ (Nr. CCCLVI). Siehe ausserdem die Beschreibung von B. Wiesmüller und das Abbildungsmaterial in der Leipziger Datenbank (zugänglich unter https://www.islamic-manuscripts.net/ content/index.xml; Stand 17. August 2017): Abb. 2-18 (vollständig beschrieben) und Abb. 19-23 (erst Streifen am Rand beschrieben); Abb. 24: Etui.

34 Vgl. Is 1625 (Dublin, Chester Beatty Library; Kapitel 4.7): Das Schriftband ist in diesem Fall einteilig. Siehe ausserdem Kopenhagen, David Collection, Nr. 37-1996 (Kapitel 4.2).

35 Vgl. Is 1626, Dublin, Chester Beatty Library (Kapitel 4.9): Das Schriftband ist in diesem Fall zweiteilig.

36 Siehe Cod. arab. 53 (Kopenhagen, Royal Library; Kapitel 4.4). Vgl. damit Michigan, Ann Arbor, Isl. Ms. 220 (zweiter, alter Teil; Kapitel 4.1): Das Schriftband ist in diesem zweiten Fall zweiteilig.

37 Wichtige Belegstücke dieses Typs sind Basel, U B, M III 173 (Kapitel 4.8); Paris, BNF, Arabe 6088 (Kapitel 4.5); Berlin, Staatsbibliothek, Ms. or. oct. 218 (Kapitel 4.3); Dublin, Chester Beatty Library, Is 1624 (Kapitel 4.6). 
mehrfach Abschnitte in mikroskopischer Schrift ( $\dot{g} u b \bar{a} r)$ kopiert und derart angeordnet worden sind, dass sie einen übergeordneten Text in grosser Schrift entstehen lassen. Stellen in $\dot{G} u b \bar{a} r$-Schrift sind zwar auch auf sämtlichen andern hier untersuchten Belegstücken vorhanden. Ihre Anordnung bildet darauf aber keinen übergeordneten Text. ${ }^{38}$

Zum $\dot{G} u b a \bar{r}$-Stellen-Typ gehören einerseits Exemplare, die in Iran angefertigt worden sind (Typ B.1). ${ }^{39}$ Das Vorgehen, einen übergeordneten Text aus Stellen in mikroskopischer Schrift zu bilden, scheint später aber auch auf dem indischen Subkontinent gepflegt worden zu sein (Typ B.2: indisch-persischer Subtyp). ${ }^{40}$ Ausserdem lassen sich Dokumente dieser Art feststellen, die zwar aus Iran selbst stammen dürften, aber auf Pergament notiert worden sind (Typ B.3). Die vorliegende Arbeit behandelt in erster Linie die mit grosser Wahrscheinlichkeit aus Iran selbst stammenden Exemplare (Typ B.1). Sie macht der Vollständigkeit halber aber auch auf die beiden andern Subtypen (B.2 und B.3) aufmerksam.

B.1: Die Breite der Dokumente des persischen Typs B.1 beträgt ca. $10 \mathrm{~cm}$. Ihre Länge ist mit 6 $8 \mathrm{~m}$ deutlich geringer als jene der Belegstücke des Städtedreieck-Typs. ${ }^{41}$ Die dem persichen Typ zugewiesenen Exemplare sind grob ins 16.-18. Jh. zu datieren. Sie können im Einzelfall etwas früher oder später angefertigt worden sein. Für die Herstellung solcher Dokumente wurde ab der zweiten Hälfte des 16. Jh. ein hauchdünnes, nahezu transparentes und sehr gut geglättetes Papier verwendet. Dieser Beschreibstoff unterscheidet sich deutlich von jenem, der für die Belegstücke des Städtedreieck-Typs verwendet worden ist. ${ }^{42}$ Die Klebe-

38 Einzig auf dem ersten Teil von Is 1624 (Chester Beatty Library, Dublin; datierend aus dem Jahr 1362) sind Stellen in $\dot{G} u b \bar{a} r$-Schrift ebenso derart angeordnet, dass übergeordneter Text entsteht.

Vgl. dazu Kapitel 5.1-5.

Siehe dazu die Hinweise in Kapitel 7.1.

41 Vgl. z. B.: 1. Bayani et. al., The decorated word 274-275 (Nr. 71) und 278-279 (Nr. 73). 2. Paris, BNF, Arabe 5102; aufrufbar unter: http://gallica.bnf.fr/ark:/12148/btvib84192247.r $=$ Arabe+5102. LangDE (Stand 17. August 2017).

42

stellen bei den Exemplaren mit transparentem Papier sind zumeist knapp bemessen; die Überlappungen zwischen den einzelnen Papierstreifen betragen in der Regel nur etwa $5 \mathrm{~mm}$.

Dem persischen Typ lassen sich neben Св 542, Fondation M. Bodmer, Cologny-Genève, ${ }^{43}$ weitere Belegstücke zuordnen. ${ }^{44}$ Die Rolle Ms. or. oct. 146, Staatsbibliothek, Berlin, datiert aus dem 16.Jh. und weist mehrfach mit dem vorangehenden Belegstück vergleichbare Stilmerkmale auf. ${ }^{45}$ Sie weicht aber dennoch in wichtigen Punkten davon ab (persische Gebete, figürliche Darstellungen etc.). Hierhin gehört sodann Is 1623, Chester Beatty Library, Dublin, das aus dem Jahr 1578 datiert. ${ }^{46}$ Aus Iran, und wohl aus dem 18. Jh., stammen weitere Exemplare, die trotz ihrer späteren Entstehungszeit Gemeinsamkeiten mit den bereits im 16 . Jh. angefertigten Belegstücken aufweisen. Es handelt sich u.a. um Paris, BNF, Arabe $5102,{ }^{47}$ und Dublin, Chester Beatty Library, Is 1621 und $1622 .{ }^{48}$ Die vorliegende Arbeit behandelt bei der Auseinandersetzung mit diesem Typ in erster Linie die soeben angeführten Dokumente; sie sind mit grosser Wahrscheinlichkeit in Iran selbst angefertigt worden.

B.2, indisch-persischer Subtyp: Es lassen sich aber auch Belegstücke dieses Typs nachweisen, die mutmasslich ausserhalb Irans, mit grosser Wahrscheinlichkeit auf dem indischen Subkontinent, angefertigt worden sind. Auch diese etwas später (17./18.-19. Jh.) entstandenen Dokumente weisen das vom persischen Grundtyp bekannte

und Ms. or. oct. 146 (Staatsbibliothek, Berlin) werden dem persischen Typ zugewiesen. Bei ihrer Herstellung ist allerdings ein starkes Papier verwendet worden.

Es enthält Gestaltungselemente, die sich auf Belegstücken aus dem iranischen Kulturraum häufig nachweisen lassen. Aus einem nachträglich angebrachten handschriftlichen Vermerk am Rollenanfang lässt sich schliessen, dass dieses Belegstück vor $155^{\circ}$ angefertigt worden sein muss.

45 Vgl. Kapitel 5.1.

46 Vgl. Kapitel 5.3.

47 Vgl. Kapitel 5.4.

48 Vgl. Kapitel 5.5. 
Layout-Prinzip auf (Bildung eines übergeordneten Texts durch Stellen in $\dot{G} u b \bar{a} r$-Schrift). Sie sind von geringerer Qualität und Originalität als die zuvor angeführten Dokumente aus Iran selbst. Es sprechen wiederholt auch verschiedene Hinweise dafür, dass sie aus kommerzieller Serienherstellung stammen und in spezialisierten Ateliers angefertigt worden sind. Wichtige Vertreter dieser Art von späteren Dokumenten sind einerseits Paris, BNF, Arabe 571, und ein Parallelstück dazu aus Kopenhagen, Royal Library, Cod. Arab. Add. $49 \cdot{ }^{49}$ Hierhin gehört sodann ein Exemplar aus Rom (Biblioteca Apostolica Vaticana: Ms. Vat. Arab. 882). ${ }^{50}$

$\mathrm{Zu}$ den mutmasslich ausserhalb Irans angefertigten Dokumenten dieser Art zählt ausserdem eine zweite Gruppe von zusätzlichen Exemplaren. Darauf ist jeweils der Thronvers (Q 2:255) in der geschilderten Art (grosser Text, der sich aus Stellen in $\dot{G} u b \overline{a r}$-Schrift zusammensetzt) kopiert und in mehreren Kartuschen über die gesamte Länge der Rolle verteilt worden. Sie befinden sich heute in Dublin (Chester Beatty Library), London (British Library), Oxford (Bodleian Library), Manchester (John Rylands Library) und Cambridge (Fitzwilliam Museum).51

B.3, persischer Typ auf Pergament: Stellen in $\dot{G} u b \bar{a} r$-Schrift, die einen übergeordneten Text bilden, sind ausserdem ein Merkmal für Dokumente, bei deren Beschreibstoff es sich um Pergament handelt und die wahrscheinlich in Iran selbst angefertigt worden sind. Da diese Arbeit aber nur Dokumente berücksichtigt, bei deren Beschreibstoff es sich um Papier handelt, wird hier nur der Vollständigkeit halber auf diese Belegstücke aus Pergament aufmerksam gemacht.

Diese Dokumente sind relativ schmal (ca. 4$6 \mathrm{~cm}$ ). Ausserdem bestimmt die Länge der bei der Herstellung verwendeten Tierhäute auch die Länge der Belegstücke selbst. Sie messen selten mehr als $70 \mathrm{~cm}$. Diese Art von Rollen ist wahr-

49 Vgl. Kapitel 7.1.

50 Vgl. dazu Kapitel 7.1, bei Anm. 24.

51 Vgl. dazu Kapitel 7.1, bei Anm. 24-3o. scheinlich zumeist im 19. Jh. angefertigt worden. Ihr wichtigstes Charakteristikum besteht darin, dass sie die Namen der Čahārdah Mașūm, der 14 Sündlosen, aufzählen. ${ }^{52}$ Dies ist ein deutlicher Hinweis, dass sie in einem zwölfer-schiitischen Umfeld entstanden sind. ${ }^{53}$

C. Der osmanisch-türkische Typ: ${ }^{54}$ Sein Hauptmerkmal besteht darin, dass in der zweiten Hälfte dieser Dokumente Būṣīīs (gest. zwischen 1294 und 1297) Qașïdat al-Burda in weitgehend identischer Weise kopiert worden ist. Die einzelnen Verse wurden darauf in $\dot{G} u b \bar{a} r$-Schrift in Kästchen notiert, die gegenüber der Rollenrichtung geneigt angeordnet sind. Auch lassen sich auf Belegstücken dieses Typs weitere textliche und ornamentale Ähnlichkeiten beobachten.

C.1: Zu diesem Typ zählt einerseits Ms. or. 20 aus der Zentralbibliothek Zürich. ${ }^{55}$ Dazu gehört überdies ein Belegstück aus dem Historischen Museum in Budapest. ${ }^{56} \mathrm{Zu}$ dieser Gruppe zählt ausserdem das in breiteren Kreisen bekannte Belegstück aus der Badischen Landesbibliothek in Karlsruhe. ${ }^{57}$ Ebenso hierhin gehören sodann Belegstücke aus Leipzig, Stuttgart, München und Berlin. ${ }^{58}$

Die Dokumente dieses Typs sind deutlich kürzer und schmaler als jene der Typen A.1-3 und B.1-2. Sie messen bei einer Breite von ca. $4 \mathrm{~cm}$ ungefähr 3-4 $\mathrm{m}$ in der Länge. Sie stammen in der Regel aus osmanischen Kontexten und datieren aus dem 16.17. Jh. Auch in diesem Fall wurde bei der Herstellung zumeist ein hauchdünnes Papier verwendet.

C.2: Aus dem osmanischen Kulturraum sind neben den Belegstücken mit einer Abschrift der Burda weitere Dokumente erhalten geblieben. Sie

52 Bei den 14 Sündlosen handelte es sich um Muhammad, Fāțima und die 12 schiitischen Imame; siehe Algar, Čahārdah Ma'sūm, in EIr.

53 Vgl. dazu Kapitel 7.2, bei Anm. 37-41.

54 Vgl. Nünlist, Rollen der Andacht aus dem Umfeld von Derwischorden.

55 Vgl. Nünlist, Handschriften 74-80 (Ms. or. 20).

56 Budapest, Historisches Museum, в тм ко 99.119.3.

57 Karlsruhe, Badische Landesbibliothek, RA 204.

$5^{8}$ Leipzig, Universitätsbibliothek, B. or. 328; Stuttgart, Württembergische Landesbibliothek, Cod. or. $8^{\circ} 83$; Berlin, Staatsbibliothek, Ms. or. oct. 403. 
werden hier allerdings nicht näher behandelt. Die folgende Aufzählung macht jedoch auf ausgewählte zusätzliche Exemplare aus dem osmanischtürkischen Einflussbereich aufmerksam. Ihre Zuweisung $\mathrm{zu}$ osmanischen Kontexten ist gesichert, da sie zumeist längere Textelemente (v.a. Gebete) auf Osmanisch-Türkisch enthalten. Hierhin gehören u. a. die Belegstücke Gotha, Ms. orient. T 4; Berlin, Staatsbibliothek, Ms. or. oct. 172; München, BSB, Cod. Turc. 41 und 42; Weimar, AmaliaBibliothek, Oct. 183; ${ }^{59}$ Leipzig, Universitätsbibliothek, B. or. 321, 322, 329 und Ms. Gabelentz 46 und 59; Oxford, Bodleian Library, Ms. Arab. g 4.

\subsection{Vorbemerkungen zur ideologischen Kontextualisierung}

Am Anfang der Auseinandersetzung mit den hier untersuchten Dokumenten war ihre zeitliche und geographische Einordnung weitgehend unklar. Wiederholt mussten in einem ersten Schritt ins Auge gefasste chronologische und geographische Zuschreibungen drastisch korrigiert werden. ${ }^{60}$ Für die präzisere Einordnung der Belegstücke haben sich stilistische Vergleiche als hilfreich erwiesen, wie sie auch in der Kunstgeschichte angestellt werden. Dieses Vorgehen liess häufig Rückschlüsse auf entsprechende Einordnungen zu. Die geographische Kontextualisierung wird allerdings oft durch die hohe Mobilität der an der Herstellung der Rollen beteiligten Kunsthandwerker und der fertigen Dokumente erschwert. Mit ähnlichen Fragestellungen sieht sich zwar auch der Spezialist für islamische Architekturgeschichte konfrontiert. Allerdings ist er insofern in einer komfortablen Lage, als er das fertige Bauwerk klar lokalisieren kann. Dies wiederum erleichtert ihm

59 Beschrieben in Sobieroj, Handschriften: Teil 5: Thüringen 134f. (Nr. 72).

6o So z.B. im Fall von Ms. or. 20 aus der Zentralbibliothek Zürich, das ins 17. Jh. datiert werden muss (anstatt 19. Jh.). Eine noch deutlichere Korrektur war in Bezug auf M III 173 aus der Universitätsbibliothek Basel erforderlich (um 1400, anstatt 17.-18. Jh.). den Zugang zu Archivmaterialien und historischen Dokumenten, die ihm bei der weiteren, u. a. auch ideologischen, Kontextualisierung dienlich sind.

Obwohl im Fall der Dokumente in Rollenform derartige konkrete Informationen zur Herkunft zumeist fehlen, ${ }^{61}$ haben sich die aus der islamischen Kunstgeschichte bekannten komparativen Methoden auch im vorliegenden Zusammenhang als dienlich erwiesen. Es zeigte sich, dass das aus Schreinbauten bekannte textliche und visuelle Vokabular wiederholt grosse Ähnlichkeiten mit jenem auf den Rollen aufweist. Diese Schreinbauten wiederum weisen Bezüge zur islamischen Mystik und zu den aus ihr hervorgegangenen bzw. zu den mit ihr verwandten Männerbünden auf. Jedenfalls haben sich bei der Analyse der Rollen Vergleiche mit Schreinbauten als fruchtbarer erwiesen, als das Beiziehen von Prachthandschriften des Korans oder das Untersuchen der Ornamentik grosser Moscheebauten in der islamischen Welt.

Die soeben angestellten Beobachtungen, besonders auch der Umstand, dass die auf den hier untersuchten Dokumenten enthaltenen Textelemente zumeist frommer oder erbaulicher Art sind, ${ }^{62}$ lassen Zweifel an der Berechtigung ihrer bisherigen ideologischen Kontextualisierung aufkommen. Die westliche Forschung hat sich bis anhin zwar erst ansatzweise mit den hier untersuchten Dokumenten befasst. Wenn sie es doch taten, fassten frühere Studien in erster Linie eine Erklärung dieser Belegstücke aus der islamischen Magie (sihr) heraus ins Auge.

G. Anawati hat sich in einem 1972 veröffentlichten Aufsatz mit handschriftlichen Dokumenten in Rollenform aus Mali befasst, die mutmasslich aus der ersten Hälfte des 20. Jh. stammen. ${ }^{63}$

61 Mit der Aufnahme dieser Dokumente in Bibliotheken ist zwar ihr Erhalt besser gesichert. Allerdings gehen dadurch nahezu sämtliche Informationen zu ihrer ursprünglichen geographischen, chronologischen und ideologischen Kontextualisierung verloren.

62 Der Inhalt der Rollen wird im Lauf dieser Arbeit präzisiert.

63 Anawati, Trois talismans musulmans. 
Er ordnet diese Belegstücke, die wiederholt mit den in dieser Arbeit vorzustellenden Dokumenten identische Elemente enthalten, der Magie zu. Hinzuweisen ist ausserdem auf die Untersuchungen von R. Kriss und H. Kriss-Heinrich zum Volksglauben im Islam, die gerade zum Einstieg in die Thematik grosse Verbreitung gefunden haben. Die beiden Autoren bezeichnen einfachere Dokumente dieser Art als „Zauberrollen“.64 Die Bodleian Library, Oxford, wiederum besitzt zwei Dokumente auf Pergament, die auf dem Bibliotheksbehältnis, wohl zu Unrecht, als „magical roll“ bezeichnet werden. ${ }^{65}$ Sie wurden 2013 in ihrem Behältnis verschlossen in einer Ausstellung über Magie in der Radcliffe Camera (Oxford) gezeigt. ${ }^{66}$

Für unsere Belange von Interesse sind sodann die Untersuchungen von K.R. Schaefer, der sich in einer Monographie mit im Blockdruck-Verfahren (Tarš-Technik) angefertigten Dokumenten auseinandersetzt und die darauf enthaltenen Textelemente erfasst. ${ }^{67}$ Diese Belegstücke werden in der vorliegenden Arbeit zwar nicht näher untersucht. Allerdings weisen sie aufgrund des darauf enthaltenen visuellen und textlichen Vokabulars eine grosse Nähe zu den hier im Vordergrund stehenden handschriftlichen Belegstücken auf. Damit ist auch ihre Charakterisierung als enigmatic charms durch K.R. Schaefer von Interesse. Während der Begriff enigmatic weitgehend unproblematisch ist und durch seine Bedeutung „rätselhaft“ bei der Auseinandersetzung damit vielfache Zugänge offen lässt, sind mit dem Ausdruck charm, zumal im Englischen, zahlreiche a prioris verbunden, die eine grosse Nähe dieser Blockdruck-Dokumente

64 Vgl. Kriss und Kriss-Heinrich, Amulette Abb. 98-99 (Beispiele aus Sarajewo und Mostar).

65 Bodleian Library, Oxford: MS. Arab. g. 12 (R) und 13 (R). Ebenso als „Arabic magical roll“ wird auf dem Bibliotheksbehältnis MS. Arab. g. 11 (R) aus derselben Bibliothek bezeichnet. Dieses Exemplar von einfacher Machart enthält endlose Wiederholungen der basmala (Beschreibstoff: Papier).

66 Bodleian Library, Oxford, Radcliffe Camera: „Magical Books: From the Middle Ages to Middle-earth“ (Ausstellung bis am 27. Oktober 2013).

67 Schaefer, Enigmatic charms zur Magie implizieren. ${ }^{68}$ Derartige Dokumente werden in islamischen Kontexten gemäss einer weit verbreiteten Auffassung von Magiern, Zauberern und Heilern angefertigt.

Vor dem Hintergrund der bisherigen wissenschaftlichen Arbeiten ist verständlich, dass Y. Alsaleh bereits im Titel ihrer Dissertation über solche Rollen (Licit magic) eine Zuordnung zur Magie vornimmt. ${ }^{69}$ Sie weist darauf hin, dass diese Rollen mehrfach Elemente enthalten, wie sie auch aus Handschriften magischen Inhalts bekannt sind (z.B. Buchstaben- und Zahlenquadrate, Gitter). Ihre Arbeit stützt sich einerseits auf die von K.R. Schaefer zusammengetragenen Tarš-Dokumente, zieht aber zusätzlich zwei handschriftliche Rollen aus der Dār al-ātār al-islāmiyya in Kuwait bei. Diese beiden handschriftlichen Rollen zählen zu den Dokumenten jener Art, die auch in dieser Studie untersucht werden.

Y. Alsaleh befasst sich in ihrer Arbeit eingehend mit der šarĩa-rechtlichen Stellung der Magie und erörtert die dazu vertretenen Standpunkte insbesondere anhand der Auffassungen der Ihwān aṣ-Ṣafā' (10. Jh.) und Aḥmad al-Būnīs (gest. wohl 1225). ${ }^{70}$ Sie macht zusätzlich auf die diesbezüglichen Ausführungen in Ibn an-Nadīms (gest. 995 oder 998) Fihrist und Ibn Haldūns Muqaddima aufmerksam. ${ }^{71}$ Y. Alsaleh hält dabei fest, dass Weisse Magie im Islam halāl, erlaubt, sei. ${ }^{72}$ Während diese Feststellung nicht angezweifelt werden soll, ist der Umkehrschluss allerdings nicht zulässig: Es ist nicht wirklich ersichtlich, weshalb, was haläl ist, auch magisch sein muss. Es soll allerdings nicht

68 Der Oxford English Dictionary (online) definiert charm u.a. als „a. orig. The chanting or recitation of a verse supposed to possess magic power or occult influence; incantation, enchantment; hence, any action, process, verse, sentence, word, or material thing, credited with such properties; a magic spell; a talisman, etc.“

69 Alsaleh, Licit Magic.

70 Vgl. op. cit. 16-45 (Ihwān aṣ-Șafā') und 45-78 (Būnī).

71 Vgl. op. cit. 186-189 (Ibn an-Nadīm) und 189-200 (Ibn Haldūn).

72 Mit der Frage nach dem Erlaubtsein der Magie hat sich ausführlich M. Dols befasst: Majnūn 261-310 und sein Index, s.v. magic (532). 
verschwiegen werden, dass auch Y. Alsaleh vielfach auf den frommen und erbaulichen Inhalt der von ihr berücksichtigten Dokumente hinweist. ${ }^{73}$

Mit den hier untersuchten Dokumenten in Rollenform eng verwandt sind aufgrund ihrer Kombination von gestalterischen und textlichen Elementen ausserdem Hemden aus Stoff. Es hat sich in der Forschung durchgesetzt, derartige Kleidungsstücke als Talisman-Hemden zu bezeichnen. ${ }^{74}$ Dies kommt gerade auch in den Arbeiten H. Tezcans zum Ausdruck, die sich wiederholt mit derartigen Hemden beschäftigt hat. ${ }^{75}$ Sie bezeichnet diese

73 Alsaleh, Licit magic z. B. 80-83.

74 Die uв Leipzig besitzt ein derartiges Schutzhemd (Türkenhemd, ohne Signatur), das derVerfasser am 6. Dezember 2017 untersuchen konnte; vgl. Klemm, Ein Garten im Ärmel $22 \mathrm{f}$.

Vgl. von Hammer-Purgstall, Ueber die gefeyten talismanischen [!] Hemden der Moslimen; Demonsablon, Notes sur deux vêtements talismaniques [!]; Atil, Sultan Süleyman 196-199; Maddison und Savage-Smith, Sciences, tools and magic. Part I: Body and spirit, darin 117-122 (Kapitel Talismanic shirts); Tezcan, Şifalı gömlekler; dieselbe, Tılıslımlı [!] gömlekler; Brac de la Perrière, Tuniques talismaniques [!] indiennes.

Diese Hemden sind mit grossem Aufwand hergestellt worden. Sie wurden angeblich im Krieg direkt auf der Haut getragen und dienten zum Schutz vor Verletzungen. Sie wurden u. a. in den Ateliers des Sultans in Istanbul angefertigt, sind aber ebenso aus Iran und Indien bekannt. Vereinzelt ist belegt, dass ihre Anfertigung mehrere Jahre dauerte. E. Atıl, Sultan Süleyman v.a. 196198, hinterfragt in einer Bemerkung zu einem Belegstück allerdings die angebliche Herstellungsdauer von nahezu vier Jahren. Man habe damit wohl Bewunderung hervorrufen wollen.

Die Beteiligten dürften beim Anfertigen derartiger Schutzhemden komplexe Anweisungen und Vorsichtsmassnahmen beachtet haben, wie sie u.a. in den Werken Būnīs oder Tilimsānīs festgehalten werden. J. von Hammer-Purgstall, Über die gefeyten talismanischen Hemden 2, hebt einen Hinweis hervor, wonach vierzig Jungfrauen den Stoff für ein solches Hemd anfertigten. Die an der Herstellung derartiger Schutzmittel beteiligten Spezialisten haben zumeist über umfassende Kenntnisse der Astronomie oder der besondereren Eigenschaften der unterschiedlichsten Pflanzen verfügt (vgl. E. Atıl, op. cit. 196). Sie sollen vor dem Beginn ihres opus auch die verschiedensten Massnahmen getroffen haben, damit ihre Bemühungen von Erfolg gekrönt seien (z. B. das Einhalten von Reinheitsgeboten, das Bestimmen des für das
Kleidungsstücke im Titel einer ihrer Untersuchungen als tılıslımlı gömlekler. ${ }^{76}$

Die Problematik der Begrifflichkeit „TalismanHemden“ (bzw. tılıslımlı gömlekler) - sie lässt sich aufgrund der häufig ähnlichen Gestaltung der Rollen auch auf diese übertragen - besteht darin, dass damit zahllose interpretatorische a prioris verbunden sind. Die mit ihrer Verwendung verbundenen impliziten Annahmen lenken den Blick in eine ganz bestimmte Richtung und schliessen alternative Zugänge vorschnell aus. Wer in den vorliegenden Zusammenhängen von Talismanen redet, nimmt zumindest implizit eine Zuordnung dieser Objekte zur Magie vor. ${ }^{77}$ Als unverfänglicher hat sich die Bezeichnung dieser Gegenstände als Amulette erwiesen. ${ }^{78}$ Allerdings weist auch sie noch eine grosse Affinität zu magischen Vorstellungen auf.

Die soeben angestellten Überlegungen mahnen zur Vorsicht bei der Charakterisierung der hier untersuchten Gegenstände. Es fällt auch auf, dass die Übersetzungen der auf einem solchen Hemd enthaltenen Textstellen durch J. von Hammer-Purgstall einen engen Bezug zur islamischen Magie (sihr) nicht wirklich nahelegen. Diese Textelemente sind weitgehend frommen und erbaulichen Inhalts. ${ }^{79}$ Es wäre deshalb nahe-

opus günstigen Zeitpunkts anhand von astronomischen Berechnungen). Ausserdem fällt auf, dass derartige Spezialisten häufig eine grosse Nähe zum Sufismus aufweisen. E. Atıl (op. cit. 198) hält fest, dass ein Derviş Ahmed b. Süleymān ein solches Hemd hergestellt habe (siehe dazu auch Anm. 92).

76 Indem H. Tezcan diese Hemden im Titel einer nachfolgenden Publikation als şifalı gömlekler („Heilungshemden“) bezeichnet, zieht allerdings auch sie alternative Zugänge in Betracht: Tezcan, Şifalı gömlekler.

77 Für eine umfassende Diskussion des Magiebegriffs vgl. Otto, Magie.

78 Als Referenzwerk über Amulette gilt Budge, Amulets and talismans. Savage-Smith, Science, tools and magic $132 \mathrm{f}$. (Kapitel Amulets and related talismanic objects), äussert sich zur ungefestigten Terminologie zur Unterscheidung zwischen Amulett und Talisman (in Maddison und Savage-Smith, Science, tools and magic).

79 Von Hammer-Purgstall, Über die gefeyten talismanischen Hemden der Moslimen. 
liegender, diese Hemden aufgrund ihrer Funktion und ihres Inhalts als „Hemden des Schutzes und der Andacht" zu definieren. Alternativ lässt sich auch die einfachere Benennung „Schutzhemden" in Betracht ziehen. Für die hier im Vordergrund stehenden Rollen gilt sinngemäss dieselbe Feststellung. Es würde sich demnach bei ihnen um Dokumente von Schutz und Andacht handeln. Diese alternative Bezeichnung erspart uns auch eine Auseinandersetzung mit der gerade im Bereich des Islams oft sehr polemisch geführten Debatte über die šarīa-rechtliche Stellung von Magie (sihr).

Die Einordnung der untersuchten Dokumente in den Kontext der Magie (sihr) in der bisherigen westlichen Forschung ist allerdings auch nachvollziehbar. Verschiedene Belegstücke enthalten in der Tat Elemente, die auch aus der breiten magischen Literatur bekannt sind (z.B. Brillenbuchstaben, ${ }^{80}$ Abfolgen von Buchstaben und Ziffern ${ }^{81}$ oder die als Salomonssiegel bekannte Zeichenfolge ${ }^{82}$ ). Ausserdem lassen sich vereinzelt Belegstücke nachweisen, die überdurchschnittlich viele sonst aus der Magie bekannte Elemente enthalten. Dazu zählt ein Dokument von eher untergeordneter Qualität aus der Thüringischen Landesbibliothek (Jena), das in einem persischen Kontext entstanden ist. ${ }^{83}$ Zumeist ist bei der Erklärung dieser Dokumente aus der Magie heraus jedoch Zurückhaltung angezeigt. Wiederholt hat sie sich sogar als Sackgasse erwiesen. Für diese Zurückhaltung lassen sich mindestens drei verschiedene Argumente vorbringen:

a. Das Belegstück aus Basel $^{84}$ weist auf einem Abschnitt von ca. 10-12 cm Länge Abfolgen von

8o Vgl. dazu Canaan, Decipherment 169 (Fig. 29); Winkler, Siegel und Charaktere 150-167.

81 Vgl. dazu Canaan, Decipherment 152-166 (Abschnitt III. Letters and Numbers).

82 Canaan, Decipherment, 169f. (Fig. 3oA); Winkler, Siegel und Charaktere 114-119.

83 Vgl. dazu bereits oben bei Anm. 17 und Thüringer Universitäts- und Landesbibliothek, Jena, Ms. Prov. o. 225a (beschrieben von Sobjeroi, Handschriften 54-55, Nr. 26).

84 Basel, Universitätsbibliothek, M III 173.
Ziffern und Buchstaben sowie Stellen mit Brillenbuchstaben auf. Derartige Elemente sind ähnlich auch aus dem magischen Schrifttum bekannt. ${ }^{85}$ Allerdings misst der erhalten gebliebene Teil des Basler Dokuments 8.9 $\mathrm{m}$ und seine ursprüngliche Länge könnte ohne weiteres $12 \mathrm{~m}$ erreicht haben. Es ist nicht nachvollziehbar, weshalb aufgrund des Inhalts eines Abschnitts, der gerade etwa 1 Prozent der Gesamtlänge des ursprünglichen Dokuments ausmacht, das Exemplar als Ganzes aus der Magie heraus erklärt werden soll.

b. Zweifel an einer ausschliesslichen Einordnung dieser Dokumente in magische Kontexte kommen zudem auf, da einzelne Belegstücke Angaben enthalten, wonach sie "gegen das Übel eines jeden Zauberers und einer jeden Zauberin“ wirksam seien. ${ }^{86}$ Es lässt sich zwar dahingehend argumentieren, dass hier ein Zauberer (sāhir) versucht habe, ein Schutzmittel herzustellen, das noch wirksamer ist als jenes seines Magier-Kollegen und Kontrahenten. Die Formulierung allerdings, wonach dieses Dokument gegen jeden Zauberer und jede Zaubererin („'alā kulli sāhiir wa-sāhịira“) wirksam sei, enthält doch eine Generalkritik an der Magie. Dieses grundsätzliche Hinterfragen des Handelns des sāḥir auf dem Basler Belegstück ist zwar isoliert. Es gibt aber doch Anlass dazu,

85 Basel, Universitätsbibliothek, M III 173. Vgl. Abb. 11 der Rolle unter http://www.e-manuscripta.ch/bau/content/ pageview/837978 (Stand 26. April 2017).

86 Basel, Universitätsbibliothek, M III 173: Der 4. Haykal enthält u.a. die folgenden Bitte (Zeilen 1-4: واعيذ نفسى من شر كل ساحر وساحره [...] vor dem Übel eines jeden Zauberers und einer jeden Zauberin." Vgl. Abb. 4 der Rolle unter http://www .e-manuscripta.ch/bau/content/pageview/837971; Stand 21. Dezember 2016).

Siehe auch Kapitel 4.4 (Abschnitt II.C.5, bei Anm. 128136): Ein Gebet auf dieser Rolle trägt den Titel Li-ibtāal as-sihr („Zur Entkräftung des Zaubers“).

Beachte ebenso Kapitel 4.1 (bei Anm. 92): Ein nachträglicher Eintrag auf der Rückseite des Dokuments hält fest, dass es bei der Abwehr von Zauberern und Zauberinnen nützlich sei (wa-'alā daf'šarr as-sāḥir wa-ssāhira). 
allgemeine Zweifel an der Berechtigung der Einordnung dieser Dokumente in die Magie anzubringen.

c. Diese Zweifel werden drittens dadurch bestärkt, dass sich die Erklärung mehrerer auf den Rollen enthaltener Figuren aus der Magie heraus als Sackgasse erwiesen hat. Am deutlichsten liess sich dies anhand einer unter dem Begriff Šakl-i 'ayn-i 'alī/‘Alì bekannten Figur aufzeigen. Sie liess sich erst sinnvoll erklären, als zu ihrer Interpretation gänzlich andere Ansätze als magische Vorstellungen in Betracht gezogen wurden. ${ }^{87}$

Auch R.E. Muravchick hat in ihren Auseinandersetzungen mit Schutzhemden in jüngerer Vergangenheit vor deren Charakterisierung als magic gewarnt. ${ }^{88}$ Mit dem Rückgriff auf den Ausdruck Magie mache man sich - allenfalls bloss implizit abhängig von den damit im Lauf der wissenschaftlichen Auseinandersetzungen im Westen entwickelten Standpunkten. Sie kritisiert, dass bei der Beschäftigung mit Magie in der islamischen Welt noch heute häufig auf die eigentlich überholten Darstellungen E. Douttés bzw. davon abhängige Untersuchungen verwiesen werde. ${ }^{89}$ Sie spricht im Zusammenhang mit der Verwendung des Begriffs Magie bei der Analyse der Schutzhemden von einem magic eraser. Der Ausdruck verwische die eigentlichen Zusammenhänge nur allzu oft. ${ }^{90}$ Ähnliche Vorbehalte lassen sich auch in Bezug auf die Einordnung der Dokumente in Rollenform ins Umfeld der Magie anbringen. Die magische Perspektive erweist sich auch daher als problematisch, da die auf diesen Schutzmitteln enthaltenen Textstellen diese Interpretation nicht wirklich stützen.

Die soeben angestellten Überlegungen heben hervor, dass bereits mit der Bezeichnung der untersuchten Dokumente bedeutende Weichen-

87 Vgl. dazu unten Kapitel 6 (bei Anm. 118-184) und Nünlist, Rollen der Andacht 123-127.

88 Muravchick, God is the best guardian.

89 Doutté, Magie et religion dans l'Afrique du Nord.

90 Vgl. dazu Muravchick, op. cit. 52-86 (Kapitel 2: The magic eraser). stellungen bezüglich ihrer Interpretation verbunden sind. Es erweist sich aus einer epistemologischen Perspektive als Hindernis, bei der Benennung dieser Belegstücke auf Begriffe zurückzugreifen, die gerade im Westen eine grosse Nähe $\mathrm{zu}$ magischen Kontexten aufweisen. Derartige Bezeichnungen versperren unter Umständen den Blick auf alternative Interpretationen unnötig. Die vorliegende Arbeit trägt diesen Vorbehalten durch die Wahl einer modifizierten Terminologie Rechnung. Indem hier einerseits von Schutzrollen bzw. Schutzhemden gesprochen wird, wird deren apotropäische Funktion in den Vordergrund gerückt. Im Fall der in dieser Arbeit primär interessierenden Rollen bietet sich aufgrund ihres zumeist frommen und erbaulichen Inhalts auch deren Bezeichnung als „Rollen der Andacht" an. Diese gegenüber der bisherigen Auseinandersetzung damit modifizierte Terminologie lässt bei der Interpretation insbesondere daher freiere Hand, als sie den Blick nicht von vornherein ausschliesslich in eine Richtung lenkt.

Die Feststellung wiederum, dass die auf den Rollen und Schutzhemden kopierten Texte zumeist frommen und erbaulichen Inhalts sind, ${ }^{91}$ ruft zusätzliche Beobachtungen R.E. Muravchicks in Erinnerung. Sie ist bei ihren Untersuchungen darauf aufmerksam geworden, dass mehrere der von ihr untersuchten Schutzhemden von Sufis hergestellt worden sind. ${ }^{92}$ Dies legt die Vermutung nahe,

91 Man beachte dazu die Abschriften der auf den Blockdruck-Dokumenten enthaltenen Texte bei Schaefer, Enigmatic charms 53-223. In dieselbe Richtung weisen auch Y. Alsalehs Feststellungen: Licit Magic z.B. IV, 250, 258, 266. So wurde auf LNS 25 Ms (Kuwait, Dār al-Ātār alislāmiyya) auch Būșīīs Qașìdat al-Burda kopiert. Dieser Text ist auch aus der islamischen Mystik bekannt; siehe Licit Magic, S. 16of., 179, 272.

92 Muravchick, God is the best guardian 25 (Anm. 43); 194 mit Hinweis auf Sultan Murāds Beziehungen zur Halwatiyya; 223 (Topkapı Saray 13/1177, mit Erwähnung 'Abd al-Qādir al-Ǧīlānīs; datiert 1532); 235 (Topkapı Saray 13/1133, angeblich 972/1564 durch den Derwisch Ahmad b. Sulaymān angefertigt, siehe Anm. 75. Schluss); 239 (Topkapı Saray 13/1164: 1575 vom Mawlawiyya-Derwisch Sinan Dede für Murād IıI. angefertigt). 
dass auch die Dokumente in Rollenform unter Sufis und mit ihren Auffassungen eng verbundenen Gläubigen beliebt waren.

\subsection{Zu den Abhängigkeiten zwischen Magie (sihr) und Mystik (tașawwuf)}

Es ist allerdings nicht gänzlich abwegig, dass die westliche Forschung die hier untersuchten Rollen wiederholt aus der islamischen Magie (sihr) heraus erklärt hat. Denn die engen Verbindungen zwischen den für ihre Frömmigkeit und innige Gläubigkeit verehrten Sufis und Vertretern der islamischen Magie sind bekannt. Sufis üben oft auch in der Regel den Magiern zugeschriebene Handlungen aus. Sie zeichnen sich jedenfalls durch eine grössere Offenheit gegenüber magischen Vorstellungen aus, als dies auf Vertreter der traditionalistisch orientierten islamischen Theologie zutrifft. P. Lory ist diesen innern Abhängigkeiten zwischen Magie und Mystik in einem Artikel nachgegangen: 93

P. Lory hebt einleitend definitorische Aspekte hervor und macht darauf aufmerksam, dass die Magie zu den Geheimwissenschaften ('ulüm hāfiya, ulūm bātiniyya) zähle. Der Begriff sei in der islamischen Welt erst in jüngerer Vergangenheit aus dem Westen übernommen worden und gehe letztlich auf Agrippa von Nettesheims (1486-1535) De occulta philosophia zurück. Er umfasse ganz unterschiedliche Wissensbereiche; dazu zähle neben Alchemie, Astronomie und Wahrsagekunst u.a. auch die Magie. Das Arabische kenne zur Bezeichnung dieser Einzeldisziplinen eine breite Terminologie. Der Begriff Wissenschaft werde durchaus zu Recht verwendet, handle es sich doch um klar und umfassend strukturierte Lehren. ${ }^{94}$ Wenn diese Wissenschaften als geheim charakterisiert würden, bringe dies einerseits zum

93 Lory, Soufisme et sciences occultes.

94 Für eine Übersicht über die Natur- und Geheimwissenschaften im Islam vgl. Ullman, Die Natur-und Geheimwissenschaften.
Ausdruck, dass es sich um diskrete, versteckte Lehren handle, die den breiten Massen verschlossen bleiben sollen. Anderseits ergebe sich aus dieser Bezeichnung auch, dass sie nicht Teil des allgemein anerkannten wissenschaftlichen Kanons bildeten und an den Rand des Denkbaren verwiesen würden. Der traditionalistisch geprägte Islam sei diesen Lehren mit derselben Feindseligkeit begegnet, wie dies im Westen die katholische Kirche getan habe.

Die muslimischen Theologen ('ulam $\vec{a}$ ) können die tatsächliche Existenz und Wirksamkeit magischer Praktiken (sihr) nicht in Abrede stellen, sprechen sich doch mehrere Koranstellen zu deren Gunsten aus. ${ }^{95} \mathrm{Am}$ deutlichsten lässt sich dies anhand von Moses' Begegnung mit den Magiern an Pharaos Hof aufzeigen. ${ }^{96}$ Allerdings ist alles Magische stets mit der Gefahr des Polytheismus (širk) verbunden. So empfiehlt Ġazzālì (gest. 1111) dem frommen Muslim, sich von derlei Wissen fernzuhalten. Es könne zwar nützlich sein, sei aber im allgemeinen mit vielen Gefahren verbunden. Die muslimischen Religionsgelehrten vertreten in dieser Angelegenheit allerdings keine geschlossene Position. So ermuntert z.B. Fah̆r ad-Dīn Rāzī (gest. 1209) jeden wahren Gelehrten dazu, sich um diese Wissenschaften zu bemühen. ${ }^{97}$ Gewiss ist ausserdem, dass die entscheidenden Debatten unter den Gelehrten die rasche Verbreitung dieser Praktiken unter den Muslimen bzw. das Weiterpflegen entsprechender vorislamischer Vorstellungen nicht verhindern konnten.

Zwar lässt sich im Lauf der historischen Entwicklungen wiederholt eine grosse Nähe zwischen Sufismus und Geheimwissenschaften erkennen. Allerdings handelt es sich dabei nicht um eine zwangsläufige Abhängigkeit. Ein Gelehrter kann sich durchaus mit Magie, Alchemie oder Astrologie befassen, ohne einer mystischen Bruderschaft anzugehören oder sich überhaupt für die Spiri-

95 Vgl. für eine Zusammenstellung der Koranstellen zur Magie Macdonald, Siḥr, in $E I^{I}$.

96 Vgl. Koran, Q 20:57-73.

97 Lory, loc. cit. 186 (Anm. 5), verweist auf die Übersetzung von Rāzīs Standpunkt in Lagarde, La magie arabe 87-94. 
tualität der Sufis zu interessieren. Auch lässt sich umgekehrt festhalten, dass man sich sehr wohl dem Sufismus anschliessen kann, ohne über übernatürliches Wissen oder Fähigkeiten zu verfügen. Dennoch lassen sich die engen Abhängigkeiten zwischen Sufismus und Magie nicht übersehen, die sich im Lauf der Geschichte ausgebildet haben.

Die frühen muslimischen Mystiker des 8.-9. Jh. haben als fromme Asketen gelebt. $\mathrm{Zu}$ dieser Zeit waren die Geheimwissenschaften noch deutlich als missliebige Überbleibsel aus der vorislamischen Zeit (ǧāhiliyya) zu erkennen. Selbst die Geschichtsgelehrten und Theologen konnten nur schwer zwischen dem durch göttliche Gnade gewirkten Wunder (mu'̆ǧiza, karāma) und dem Werk des Magiers unterscheiden. Der breiten Bevölkerung fiel diese Abgrenzung noch schwerer. Jedenfalls machte sie keinen Unterschied zwischen diesen beiden Wirkungsweisen, also zwischen mưğiza und karāma einerseits und siḥr anderseits.

Historisch betrachtet ist die Entwicklung komplex. Es lässt sich festhalten, dass die Abgrenzung zwischen den reinen Mystikern und den Spezialisten für Geheimwissenschaften vage ist. Jedenfalls sind den Sufis bereits im 9. Jh. unterschiedliche Wundertaten zugeschrieben worden. Da die grossen Mystiker aber darum bemüht waren, in Einklang mit dem traditionalistisch orientierten Islam zu leben und keine prophetischen Würden beanspruchten, betonten sie den Unterschied zwischen múğiza (Machtwunder, Prophetenwunder) und karāma (Huldwunder). ${ }^{98}$ Allāh gewährt die Huldwunder seinen frommen Dienern (wali, $\mathrm{Pl}$. awliya ') punktuell.99

Diese Vorbehalte konnten allerdings nicht verhindern, dass sich die Abgrenzungen zwischen

98 Zur Unterscheidung vergleiche auch Meier, Sufik und Kulturzerfall 107; ders., Zur Biographie Ahmad-i Ğām's 162 f.; ders., Naqšbandiyya. II. Kraftakt und Faustrecht 249; Schimmel, Mystische Dimensionen $292 \mathrm{f}$. Siehe auch folgende Anm.

99 Vgl. dazu Sarrāğ, Schlaglichter über das Sufitum 449-465 (Gramlich); weitere Quellenverweise bei Lory, op. cit. 187 (Anm. 3).
Mystik, übernatürlichen Phänomenen und den Geheimwissenschaften v.a. im Bewusstsein der breiten Bevölkerung verwischten. P. Lory jedenfalls hält fest, dass die anfänglich verpönten magischen Praktiken und Wahrsagekünste nach und nach an Wertschätzung, ja sogar Heiligkeit, gewannen. Unter den Sufis setzt sich die Auffassung durch, dass sich im Wirken des Heiligen (hier: walī, Pl. awliy $\bar{a})$ das Tun und Handeln des Propheten verlängere. Auch gilt der wal̄ als Bewahrer eines verborgenen Wissens, das Muhammad auf esoterische Art und Weise nur auserwählten Schülern habe zukommen lassen. Nur dieser kleine Kreis von Adepten habe den eigentlichen Sinn des Islams erfasst. Vor diesem Hintergrund lassen sich die Geheimwissenschaften als Teil dieses esoterischen Wissens erklären und gewinnen somit heilige Legitimität. Gemäss dieser Sichtweise hätten die Engel dieses Wissen den bedeutenden Gesandten offenbart. Die Sufiheiligen (walī) wiederum hätten das Privileg und die Pflicht, dieses Wissen zugunsten der breiten Bevölkerung auszuüben. ${ }^{100}$ Es zeigt sich hier, dass das okkulte Handeln und Wissen nicht mehr aus einem willkürlichen Akt Gottes resultieren, sondern dem Adepten im Rahmen eines Initiationserlebnisses verliehen werden. In diesem Rahmen verleiht Gott seinem Schützling auch die baraka (Segenskraft).

Zum Verständnis der Bedeutung der Geheimwissenschaften im Islam ist ausserdem auf die besondere Rolle der Schia bei deren Überlieferung zu verweisen. Wenn die schiitischen Imame auch ihrer politischen Macht verlustig gegangen waren, setzte sich in Iran und Iraq ab dem Ende des 1./7. Jh. doch die Auffassung durch, dass sie über esoterisches Wissen verfügten, das in Geheimschriften festgehalten ist. Es umfasste neben der Wahrsagekunst u.a. auch die Magie und Alchemie. So soll Ğafar aṣ-Ṣādiq (6. Imam, gest. 765) eine bedeu-

100 Idris (Henoch) wird mit der Alchemie in Verbindung gebracht. Yūsuf beherrschte die Traumdeutung (Oneiromantie). Salomon wiederum galt als Bezwinger der ğinn, und Jesus war für seine Heilkünste geschätzt. Vgl. Lory, op. cit. 188. 
tende Rolle bei der Entstehung des Ğābir b. Hayyān zugeschriebenen Korpus über Alchemie gespielt haben. ${ }^{101}$ Die ausserordentlichen Kenntnisse der Imame und ihre Fähigkeiten waren nicht einfach das Resultat eines göttlichen Gnadenakts, sondern erklären sich unmittelbar aus ihrer Rolle als Vertreter göttlicher Macht auf Erden. Diese aus der Schia bekannten Auffassungen fanden im Lauf des 3./9. Jh. Aufnahme in den sunnitisch geprägten Sufismus. Die grossen Sufimeister verstanden ihre Rolle zunehmend als jene der Nachfolger des spirituellen Wirkens des Propheten.

P. Lory erläutert die Ambiguität zwischen Magie und Sufismus in der frühen Zeit (3.-4./9.-10. Jh.), indem er auf das Schicksal Ḥallāğs (hingerichtet 922) verweist. Seine Lehre war im Vergleich mit den Positionen anderer Mystiker eigentlich nicht besonders radikal. Allerdings betätigte sich Ḥallāğ im Gegensatz zu andern Sufis auch als Prediger und Wunderwirker. Er konnte Gedanken lesen, Krankheiten heilen oder Nahrung im Überfluss aus seinem Ärmel hervorzaubern. Es war in erster Linie sein wundersames Wirken, das ihm zum Verhängnis wurde. ${ }^{102}$

Die Situation erfuhr ab dem 6./12. Jh. eine grundlegende Veränderung, als sich die Sufis in Bruderschaften (țariqa, Pl. țuruq) zu organisieren begannen und ihren sozialen Einfluss ausbauten. ${ }^{103}$ Diese Bruderschaften legitimierten sich durch Überlieferungsketten (silsila), die im Idealfall auf Muhammad zurückgehen. Sie entwickelten eine spirituelle Hierarchie, an deren Spitze der quțb (Pol) steht. Die Angehörigen dieser spirituellen Hierarchien (wali, Pl. awliy $\left.\bar{a}^{\prime}\right)$ leiteten die weltlichen Angelegenheiten in Übereinstimmung mit den göttlichen Anweisungen. Sie legten für die Menschen Fürsprache ( $\check{s} a f a \bar{a} a)$ ein und wandten den göttlichen Zorn kraft ihrer Heiligkeit von ihnen ab. Die Sufitexte weisen darauf hin, dass diese Heiligen zurückgezogen oder sogar gänzlich

101 Vgl. die Hinweise bei Lory, op. cit. 188.

102 Vgl. die Quellenverweise bei Lory, op. cit. 189 (Anm. 1).

103 Vgl. Trimingham, Sufi orders 1-30; siehe ausserdem Knysh, Islamic mysticism 169-179, v.a. 172-179; danach folgen bei Knysh Hinweise auf einzelne țarīqas. incognito lebten. Ihre Anhänger betrachteten nun aber die grossen Sufimeister als Pol (quț) oder zumindest als einen von dessen Beisitzern.

In einem derartigen Verständnis des spirituellen Lebens liess sich das Ausüben der Geheimwissenschaften durch die grossen Meister auf natürliche Art als Resultat ihrer Initiation und des dabei erhaltenen Wissens erklären. Es stellt auch einen positiven Aspekt des Wirkens der awliy $\vec{a}^{\prime}$ Gottes dar. Dieses Phänomen entfaltet in Krisensituationen eine bedeutende Wirkung.

Dieses charismatische und thaumaturgische Wirken im Umfeld der Sufibruderschaften trug massgeblich zum sozialen Zusammenhalt angesichts der mannigfaltigen Erschütterungen der muslimischen Gesellschaften im Nachgang des Mongolensturms bei (Fall Alamuts: 1256; Fall Bagdads: 1258). In diesem Zusammenhang spielen überdies die Wanderbewegungen von Derwischen aus Zentralasien nach Anatolien eine Rolle. Mehrfach ist darauf hingewiesen worden, dass die Vorsteher der Derwischorden eine bedeutende Rolle bei der allmählichen Islamisierung der turkomongolischen Einwanderer und der Bewohner der zentralasiatischen Steppen spielten. ${ }^{104}$ Es zeigt sich, dass der volkstümliche Sufismus der Derwische die Integration des magisch geprägten Brauchtums aus dem Schamanismus, wie er sich gerade in Zentralasien noch lange halten konnte, in einen vermehrt traditionalistisch orientierten Islam erleichterte. ${ }^{105}$

Vor diesem Hintergrund haben okkulte Praktiken Eingang ins Leben der Bruderschaften gefunden. Es mag zwar naheliegend sein, zwischen dem magischen Handeln eines richtigen Sufis und jenem eines Berufsmagiers zu unterscheiden. In der Realität lässt sich diese Unterscheidung aber kaum vornehmen, beriefen sich doch zahlreiche professionelle Magier und Wahrsager auf Überlieferungen aus dem Umfeld der Sufis. P. Lory illustriert dies anhand des Überlieferungsstrangs, auf den sich der bekannte Magier Aḥmad al-Būnī

104 Kreiser und Neumann, Kleine Geschichte der Türkei 61f.

105 Lory, op. cit. 19 o. 
(gest. 1225) in seinen Ausführungen zur Buchstabenmagie und zur talismanischen Magie am Ende seiner Abhandlung Šams al-ma‘āiff stützt. Er enthält die Namen der grossen Vertreter der Religion (pensée religieuse) und der islamischen Mystik. Allerdings fehlen Hinweise darauf, dass Būnī selbst Mitglied eines mystischen Ordens war. ${ }^{106}$

Für die breite Bevölkerung war es sodann ein untrügerisches Merkmal, dass jemand zu den auserwählten $a w$ liy $\bar{a}$ zählt, wenn er auch über okkulte Fähigkeiten verfügt. Es bestand grundsätzlich die Möglichkeit, auch ohne Initiation durch einen menschlichen Meister den Status eines Heiligen zu erlangen. Beim Initiator kann es sich in diesem Fall um einen Propheten, einen bereits seit langem verstorbenen Sufi oder einen Engel handeln, der dem Adepten im Traum oder in einer Vision erscheint. Diese Möglichkeit trägt zusätzlich zur Verwirrung bei und lässt die Abgrenzungen zwischen Sufismus und Magie als durchlässig erscheinen.

Die eigentlichen okkulten Wissenschaften (z.B. Astrologie, Anfertigen von Talismanen, Alchemie) waren in dieser Hinsicht in ganz unterschiedlichem Ausmass betroffen. Ohnehin bezogen sich die Anfragen der Angehörigen aus der einfachen Bevölkerungen v.a. auf ihre Sorgen im Alltag. Dazu zählen das Heilen körperlicher Krankheiten, materielles Wohlergehen und Schwierigkeiten in der Liebe. Es zeigt sich somit auch, dass die Sufimeister v.a. mit dem Anfertigen von Talismanen und Vorhersagen der Zukunft befasst sind. Die angewandten Verfahren unterscheiden sich mit der fortschreitenden Islamisierung von den aus der antiken Magie übernommenen Vorstellungen. Die koranische Offenbarung gewinnt im Rahmen der gepflegten Praktiken an Bedeutung. ${ }^{107}$

106 Lory, op. cit. 190 (Anm. 2), mit Verweis auf seinen Artikel La magie des lettres dans le Shams al-macāif d' al-Bûnî $97 \mathrm{ff}$. Man beachte auch, dass Būnī mehrfach Läuterungsriten beschreibt, wie sie gerade aus dem Sufismus bekannt sind (La magie des lettres 105).

107 Lory, op. cit. 192. Gerade auch C. Hamès spricht in seinen Arbeiten von einem usage talismanique des Korans. Er meint damit, dass Zitate aus dem Koran die Schutzwertigkeit der damit versehenen Objekte erst begründen bzw. bedeutend erhöhen. Auch er rückt
Zwar kommt dem unter dem Begriff Marabutismus aus Nordafrika und dem Sudan bekannten Phänomen im Zusammenhang mit den hier im Vordergrund stehenden Dokumenten in Rollenform keine Bedeutung zu. Da sich aber im Marabutismus die Synthese zwischen Magie und Sufismus besonders gut ausbilden konnte, sei hier dennoch auf dieses Phänomen hingewiesen: Der Sufimeister, der seine Schüler auf dem Pfad (țarīqa) zur Vereinigung mit Gott anleitet und ihnen Riten, Gebete und asketische Übungen aufträgt, ist in diesem Umfeld zugleich Hersteller von Talismanen, Heiler und Wahrsager. Ausserdem wirkt er als Schiedsrichter, Vorsteher seiner Gemeinschaft und übt häufig politischen Einfluss aus. Oft fühlen sich ihm nicht nur seine eigentlichen Schüler zu Gehorsam verpflichtet, sondern ganze Regionen und soziale Gruppierungen schliessen sich ihm an. Diese Integration der Magie in die Religion, die Mystik und das Sozialleben beschränkt sich bei weitem nicht auf das muslimische Afrika. Sie lässt sich in modifizierter Form auch anderswo beobachten. In den Augen der breiten Massen saugt der Sufiheilige, der das im Koran offenbarte göttliche Wort oder auch Gebete unablässig wiederholt, die göttliche baraka in sich auf. Dank seiner Heiligkeit (walāya) verfügt er über ausserordentliches Wissen und kann Wunder wirken. Vor diesem Hintergrund erstaunt nicht, dass man sich ihn zum religiösen Vorbild oder politischen Vorsteher aussucht. Diese Abhängigkeiten sind auch im Rahmen der vorliegenden Untersuchung relevant.

P. Lory schliesst seine Überlegungen, indem er dem Ausüben okkulter Praktiken im Umfeld des Sufismus die drei folgenden Bedeutungen zuschreibt:108

a. Aus sozialer Perspektive hat das Wunderwirken der Mystiker das kollektive Vorstellungsvermögen im Volksislam massgeblich beeinflusst. Der Sufiheilige setzt sich für den Aus-

derartige Objekte damit in die Nähe von magischem Gedankengut. Hamès, Coran et talismans.

108 Lory, op. cit. 193 f. 
gleich, manchmal sogar die Rache, des kleinen Mannes ein, der unter der Härte seines Schicksals leidet und sich mit dem Unverständnis der Herrschenden für seine Schwierigkeiten konfrontiert sieht. Der Sufiheilige verteilt auf wundersame Weise Nahrung, heilt die schlimmsten Krankheiten, befördert den ungerechten Herrscher zu Tode, indem er ihm ein einziges Wort entgegenschleudert oder einen bösen Blick zuwirft. Wenn dieser Heilige auch über schier unglaubliche Macht verfügt, lebt er doch in Armut.

b. Für die Sufis selbst, die ihrer Bruderschaft in unterschiedlicher Form verbunden sind, sind das übernatürliche Wissen und die Machtfülle ihres Meisters der unwiderlegbare Beweis dafür, dass der mystische Pfad Zugang zu andern Dimensionen des Daseins vermittelt. So lassen sich die aus der Rifāiiyya bekannten Kasteiungen dahingehend verstehen, dass die subtile Geistigkeit die feste Körperlichkeit besiegt hat. ${ }^{109}$

c. Gemäss den Darstellungen in schriftlichen und mündlichen Quellen hat die aus der Kenntnis der okkulten Gesetze resultierende Mächtigkeit für die Sufimeister selbst noch eine zusätzliche Dimension. Kraft seiner Heiligkeit befindet sich der šayh in einer paradoxen Situation: Er ist einerseits in der Lage, uneingeschränkt Wunder zu wirken. Er kann nach Belieben den Tod herbeiführen oder zum Leben erwecken und sich zugleich gänzlich dem göttlichen Willen unterordnen. Für ihn ist jeglicher Gegensatz aufgehoben. Ebenso wie das individuelle Bewusstsein des Sufis im Zustand der mystischen Vereinigung in Gott aufgeht, ebenso stellen sein Wille und seine Macht die strikte Verlängerung des Willens und der Macht Gottes dar. Er hat somit Anteil an der Fortsetzung des gött-

109 Die Angehörigen der Rifā'iyya sind dafür bekannt, dass sie bei ihren ekstatischen Riten z.B. über das Feuer gehen, glühende Kohlenstücke verschlucken oder sich Spiesse in den Körper stecken. lichen Schöpfungsakts. Der Sufimeister ist in einem gewissen Sinn das Organ Gottes auf Erden. P. Lory schliesst seine Ausführungen mit der Bemerkung, dass die Magie unter diesen Voraussetzungen keine Hilfswissenschaft mehr darstellt, die ausschliesslich nützliche, aber moralisch fragwürdige Ziele verfolgt. Sie wird vielmehr zu einem der Hauptmittel, durch die Gott die Welt verändert. 110

Für die vorliegende Untersuchung sind P. Lorys Überlegungen insofern relevant, als sie die grosse Nähe von Magie und Mystik hervorheben. Sie sollen im vorliegenden Kontext dahingehend modifiziert werden, dass sich mystisch orientierte Gruppierungen durch eine grössere Offenheit gegenüber magischen Vorstellungen auszeichnen, als dies auf andere Kreise, insbesondere die traditionalistisch orientierten Religionsgelehrten, zutrifft. Jedenfalls legen es die Resultate der vorliegenden Arbeit nahe, dass die Dokumente in Rollenform primär im Umfeld mystischer Gruppierungen und mit ihnen verwandter Organisationen entstanden sind. Diese Gruppierungen zeichnen sich oft durch heterodoxe Neigungen aus. Die entsprechenden Kreise werden später präzisiert.

Vor dem Hintergrund dieser Überlegungen erklärt die vorliegende Untersuchung die untersuchten Dokumente in erster Linie aus Auffassungen heraus, die in mystisch orientierten Kreisen und weiteren mit ihnen verwandten Gruppierungen vertreten wurden. Sie tut dies in bewusstem Gegensatz zur bisherigen westlichen Forschung. Im Vordergrund stehen Bezüge zu Derwischorden, Futuwwa-Bünden, Ahī-Gruppierungen oder den später daraus hervorgegangenen Handwerksgilden (șinf, Pl. aṣnāf). Diese Umfelder zeichneten sich durch ihre grössere Offenheit gegenüber Vorstellungen aus, die im traditionalistisch geprägten Islam verpönt waren. Dies trifft gerade in Bezug auf die Magie zu. Eine vorwiegende Erklärung der

110 Lory, op. cit. 194. 
hier zu untersuchenden Dokumente in Rollenform aus der islamischen Magie heraus, liefe aber darauf hinaus, den sekundären Zugang auf Kosten ihrer primären Funktion als Dokumente von Schutz und Andacht in den Vordergrund zu rücken. 